\title{
A MATLAB/SIMULINK Approach for a First Course in Power Electronics
}

\author{
D. Pylarinos ${ }^{1}$, I. Androulidakis ${ }^{2}$, K. Siderakis ${ }^{1}$ and E. Drakakis ${ }^{1}$ \\ ${ }^{I}$ Department of Electrical Engineering, Technological Educational Institute of Crete, Greece \\ ${ }^{2}$ University of Ioannina, Greece
}

Received 30 June 2015; Accepted 25 January 2016

\begin{abstract}
This paper describes an education scenario for a first course in power electronics based on a MATLAB/SIMULINK approach. This scenario was followed in the Electrical Engineering Department of the TEI of Crete in 2014 and students were asked to fill in a questionnaire after completing the class. The scenario was based on thyristor-based rectification and $\mathrm{AC}$ control, as prior approaches were based on the use of hardware and/or the simulation of such circuits in OrCAD/PSpice. Fourier/harmonic analysis through the use of MATLAB was added and students were encouraged to undertake projects focused on simulating other types of converters in SIMULINK to receive a passing grade. The course's syllabus, the general teaching approach and the questionnaire's results are briefly presented and discussed in the paper.
\end{abstract}

Keywords: MATLAB, SIMULINK, SimPowerSystems, power electronics, thyristor, rectifier, controller, harmonics, education.

\section{Introduction}

Modern energy conversion is a field that combines theoretical and practical knowledge from various fields that were not that interacting in the past. Further, power electronics are employed in a huge variety of applications and thus, such a course needs to balance between different and often contradicting needs in a limited time-period. In Greece, Technological Education Institutes are part of higher Education and their Electrical Engineering departments generally aim to offer a more application oriented high standard technological education and training in the field. Therefore, providing basic theoretical knowledge along with promoting the use of multi-objective engineering software is a basic goal. This paper describes an education scenario for a first course in power electronics based on the use of MATLAB/SIMULINK. This scenario was followed in the Electrical Engineering Department of the TEI of Crete in 2014 and students were asked to fill in a questionnaire after completing the class. Results are also presented as a tool to evaluate the course

There are several software in the market of power electronics simulation. Probably the most usual case is the use of PSpice, usually via the OrCAD suite [1], and PSIM [2]. Further, several other software are available with a variety of characteristics (e.g. listings in [3-5] with the addition of newer software such as GeckoCircuits [6] and PLECS [7]).

MATLAB/SIMULINK provides an alternative through the use of the SimPowerSystems toolbox [8] for SIMULINK. However, it has not managed yet to achieve an extended spread in the power electronics/circuit design\&simulation community. The limited literature available is usually focused in solving the differential equations deriving from circuits using MATLAB (e.g. [9]) whereas the extended use of SIMULINK only recently started gaining further interest (e.g. [10-11]). This should probably be attributed to the fact that MATLAB/SIMULINK is a general-purpose software whereas the alternatives are usually specially designed for circuit simulation (e.g. PSpice), and circuit designers/electronic engineers are more familiar with this approach.

However, the situation is exactly the opposite for an electrical engineer as the variety of applications makes MATLAB/SIMULINK a rather useful and powerful tool in his profession. Therefore, in 2014, it was decided to employ MATLAB/SIMULINK for the laboratory part of this course. Prior approaches included the use of hardware to perform one-phase and three-phase thyristor-based rectification and AC control (see Fig. 1) and/or the simulation of such circuits in OrCAD. Therefore, it was decided to keep the same basic schedule (focused on thyristor based rectifiers and $\mathrm{AC}$ controllers), in order to ease the transition (as failed students are allowed to repeat the course), with the addition of Fourier/harmonic analysis. Students were however encouraged to undertake projects focused on simulating other types of converters in SIMULINK to receive a passing grade. Further, a 60 question questionnaire was handed out to students that completed the course in order to gain an insight to the course and audience. The course is described in this paper along with some of the results of the questionnaire.

\footnotetext{
*E-mail address: dpylarinos@yahoo.com

ISSN: 1791-2377 @ 2016 Eastern Macedonia and Thrace Institute of Technology. All rights reserved.
} 


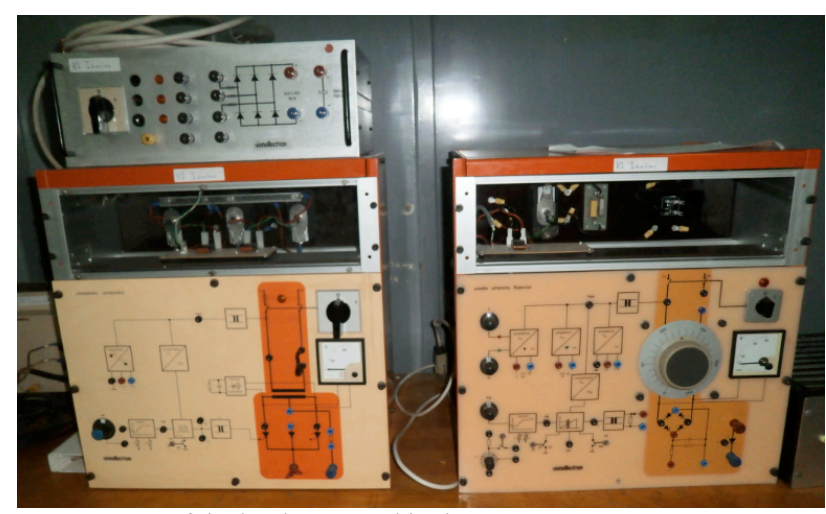

Fig. 1. Some of the hardware used in the past

\section{The course's syllabus and teaching approach}

The course included 10 different laboratory exercises, each exercise consisting of two teaching hours (45 minutes for each hour). At week 11, students were asked to fill a 60 questions questionnaire and a free discussion upon the syllabus and the approach followed. At week 12, the exam was held and the course was completed. As this was a course in the winter semester, projects were assigned on a voluntary basis before Christmas so that students would have enough time to work on them during their break. These projects were focused on the simulation of other converters (DC/DC and $\mathrm{DC} / \mathrm{AC}$ ) and indeed several students managed to complete their projects in a satisfactory manner before the exams were held. This clearly shows that the software knowledge provided to them during the course, allowed them to simulate other circuits.

The basic goal set during the planning of the course was to ease the student learning process. Instead of a syllabus based on lengthy get-to-know-the-software hours and then repetitive application, it was decided to keep the software learning in a need-to-know basis and focus on an objectoriented approach trying to maintain a high level of interest for the students. This means that students were instructed to use the software to familiarize with complex issues (such as harmonic analysis) and then turn to circuit simulation as fast as possible. In circuit simulation, new blocks were gradually discussed and used by the students each week.

The course's syllabus is mapped as follows:

Week 1: Matlab fundamentals: introduction to MATLAB, numeric operators, variables, arrays and tables, dot operator, sampling, plotting, constructing sinusoids, area in a graph, RMS value of non-sinusoid graphs (half-wave diode rectification example).

Week 2: harmonics and Fourier analysis: use of $\mathrm{fft}$ to compute the amplitude and angle spectrums (including scaling), RMS value of a current that carries harmonics, mean (real) power when only the current carries harmonics, distortion power factor and real power factor, THD

Week 3: Simulink fundamentals: introduction to Simulink, toolboxes and libraries, file types and names, SimPowerSystems, the powergui block, simulation of basic DC circuits, the current measurement block, the voltage measurement block, the display block, simulation of basic
AC circuits, the Scope block, the RMS block, simulation configuration parameters, send data to MATLAB, the mux block, the demux block, the multimeter block, fft analysis using the powergui block

Week 4: Diode rectifiers: the diode block, snubbers, the measurement port, stiff solvers, important values for rectifiers (DC and RMS values of load current-votlagepower, efficiency, form factor, ripple factor, TUF, crest factor), diode rectifier, the linear transformer block, the mean block, setting the switches as ideal from powergui

Week 5: 1-pulse rectifier: the thyristor block, the pulse generator block, signal labels, multiple axes in a graph, thyristor voltages, the product block, the divide block, calculate RMS and mean values in SIMULINK, calculate Pdc and Pac in SIMULINK, calculate efficiency, R load, RL load, R-L-Ed load, free wheel diode,

Week 6: 2-pulse rectifier: $\mathrm{R}$ load, $\mathrm{R}-\mathrm{L}$ load, continuous and non-continuous conduction, RMS and DC values in SIMULINK, load current waveform in the continuous conduction mode as inductance increases, thyristor voltages, calculate TUF

Week 7: 4-pulse rectifier: the GoTo block, the From block, $\mathrm{R}$ load, R-L load, continuous and non-continuous conduction, load current waveform in the continuous conduction mode as inductance increases, form factor, ripple factor, thyristor voltages, commutation

Week 8: 3-pulse rectifier: the 3-phase system, plotting the three phases, the 3-pulse rectifier with $\mathrm{R}$ load, continuous and non-continuous conduction, the MinMax Running Resettable block, the Abs block, calculating the crest factor in SIMULINK, calculating the Peak Inverse Voltage in SIMULINK, RMS and mean values, the Add block, thyristor voltages, portraying polar voltages, phase voltages and thyristor voltages in the same graph, R-L load

Week 9: 6-pulse rectifier: plotting all polar phases, the Synchronized 6-Pulse Generator block, the Universal Bridge block, the Gain block, creating Subsystems, R load, R-L load, continuous and non-continuous conduction, the Controlled Current Source block, snubber activation, current source load, commutation

Week 10: AC controllers: single phase with different loads, the Transport Delay block, three phase AC controller with R load, plotting all polar phases with a 0.5 gain and all phases in the same graph to explain the waveforms of phase currents

Students were asked in each Simulink exercise to simulate the circuit, calculate certain values (usually different in each exercise) and produce certain graphs. An image of the circuit for the 10th exercise is shown in Fig. 2. The instructor used a projector to illustrate the circuit, each new block, the parameters etc. These notes were available to the students after each exercise.

Occasionally signals were moved to MATLAB and plotted versus the angle in degrees (instead of seconds that simulink uses as a default), as shown in Fig. 3, so as to be more easily understood. In other cases, multiple waveforms were plotted in one scope and images were denoted, in order to better demonstrate certain phenomena (e.g. Fig. 4). 


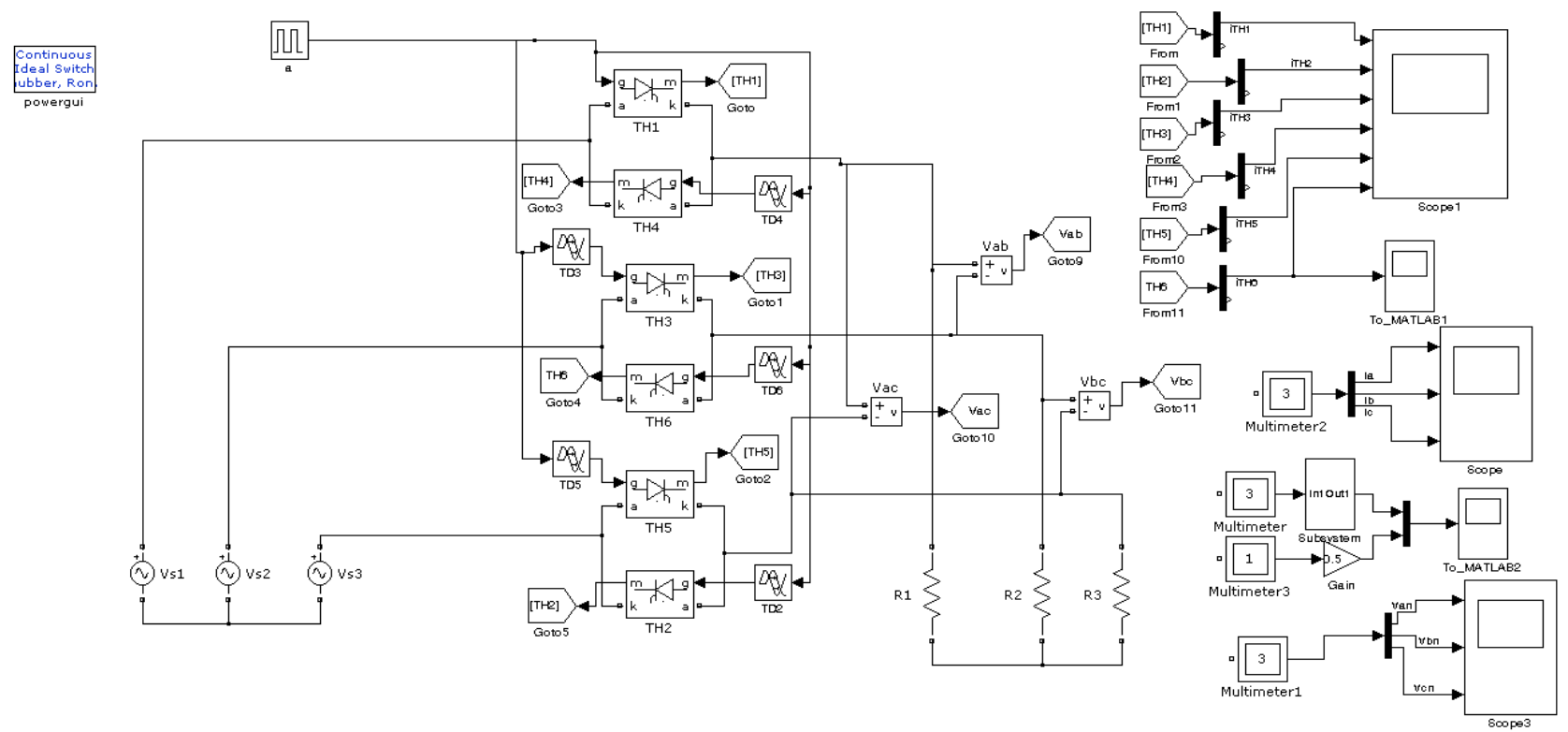

Fig. 2. An example circuit for a three phase AC controller including added graphs (e.g. phase-polar voltages)

(a)

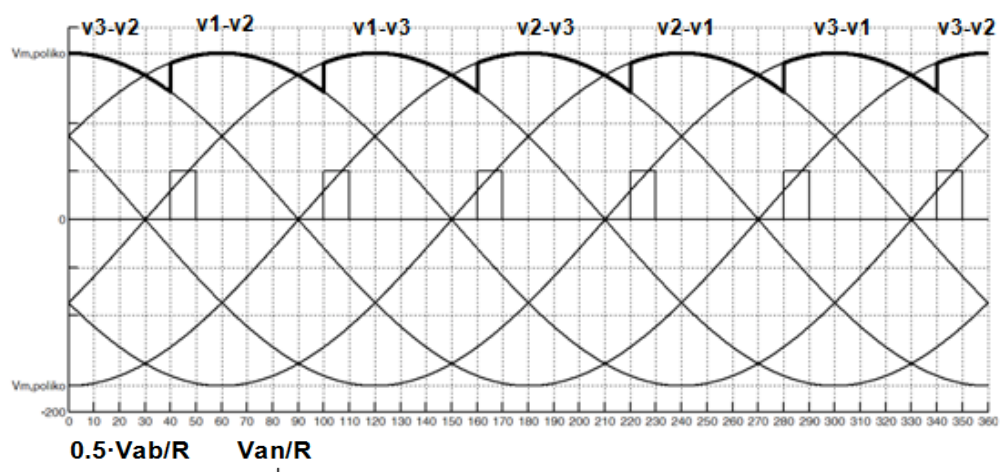

(b)

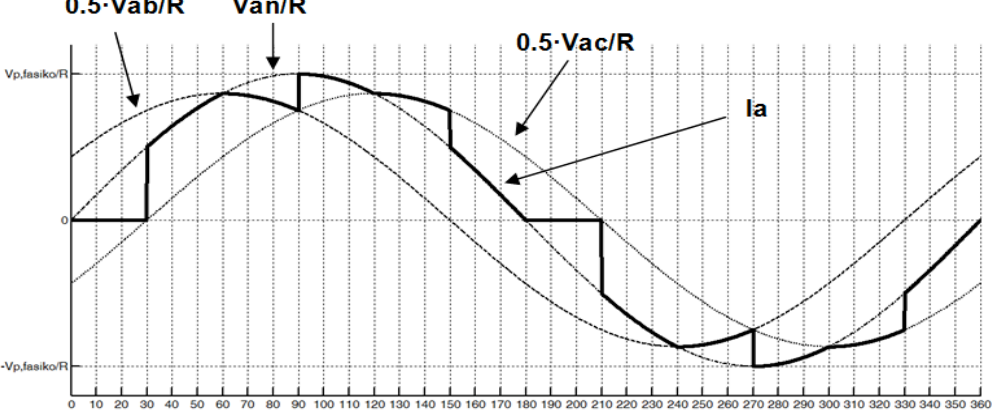

Fig. 3. Waveforms moved to MATLAB to illustrate them versus degrees. Multiple waveforms used for an improved visual representation: (a) load voltage of a 6-pulse rectifier with an R load and a firing angle of $10^{\circ}$ and (b) phase "a" current for a three phase AC controller with R load and a firing angle of $30^{\circ}$ (circuit shown in Fig. 2).

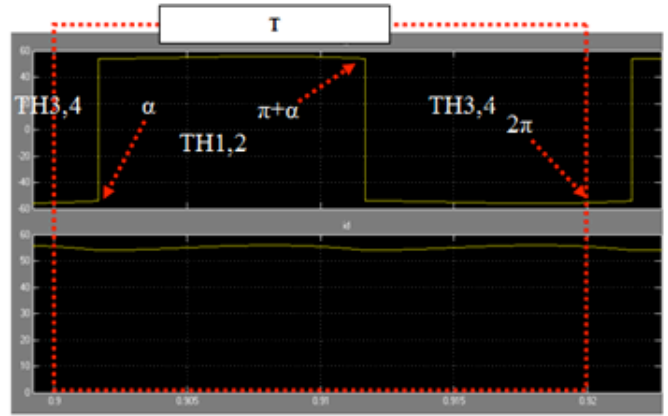

(a)

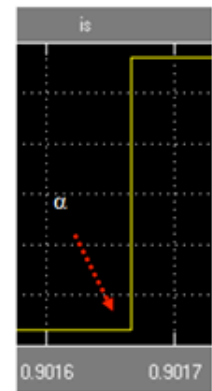

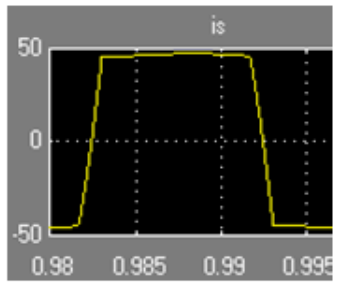

(b)

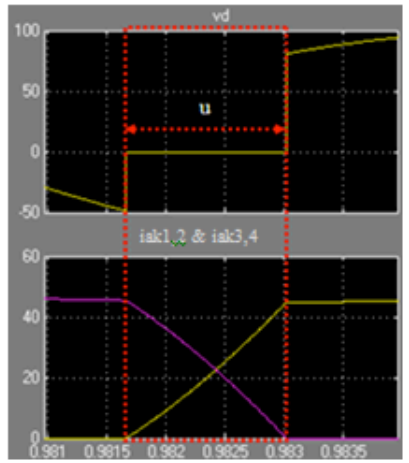

Fig 4. Graphs used to demonstrate the commutation phenomenon in a 4-pulse rectifier (a) waveforms of source current and load current if the source is considered ideal (no commutation) (b) waveforms of source current, thyristors' currents and load voltage if a source inductance of $\mathrm{Ls}=0.001 \mathrm{H}$ is considered 


\section{Questionnaire results}

A questionnaire of 60 questions was handed to the students that took this course. They were asked to answer each question by selecting a Likert-based scale from 1 to 5 , with 1 being the minimum and 5 being the maximum. Extended analysis is rather lengthy but some of the main results are mentioned below.

Results show that students consider that they have significantly improved their knowledge of MATLAB (answers 3-5: from 38\% to 98.33\%), SIMULINK (answers 3-5: from $11.67 \%$ to $96.67 \%$ ) and SimPowerSystems (answers 3-5: 3.33\% to 96.67\%)) (Fig. 5). These answers also show that students were less comfortable with PSpice (answers 3-5: 10\%) compared to MATLAB/SIMULINK, so the decision to move to MATLAB/SIMULINK is further justified.

Regarding the comprehension of basic EE topics (RMS $\&$ mean values, power, three phase system, behavior of different loads, harmonic analysis, power factors, sampling, ODE solvers etc) results show that they were significantly enhanced (answers 3-5: from 46.67\% to 95\%). The interest towards power electronics was also significantly enhanced (answers 3-5: from 30\% to 88.33\%). The course judged as satisfyingly interesting in general (answers 3-5: 90\%). It should also be noted that most students had zero or very limited knowledge/experience with power electronics (answers 3-5: 15\%). Details are shown in Fig. 6.

Other results show that the interest towards the software used was also significantly increased (answers 3-5: jumped from $33.3 \%$ to $86.6 \%$ for MATLAB and from $16.6 \%$ to $88.3 \%$ for SIMULINK), that students considered this course to be helpful for other courses (answers 3-5: 71.56\%), their diploma thesis (answers 3-5: 65\%) and for simulating other circuits in the future (answers 3-5: 95\%). Further, students declared that they would be interested to take a course focused on the application of such circuits (answers 3-5: $71.67 \%$ ) but even more interested to take a course focused on other types of converters (DC/DC and DC/AC) (answers 3-5: 83.33\%). An improvement on their level of English (technical terms) was also evident from the results (answers 3-5 jumped from $73.33 \%$ to $86.67 \%$ ).

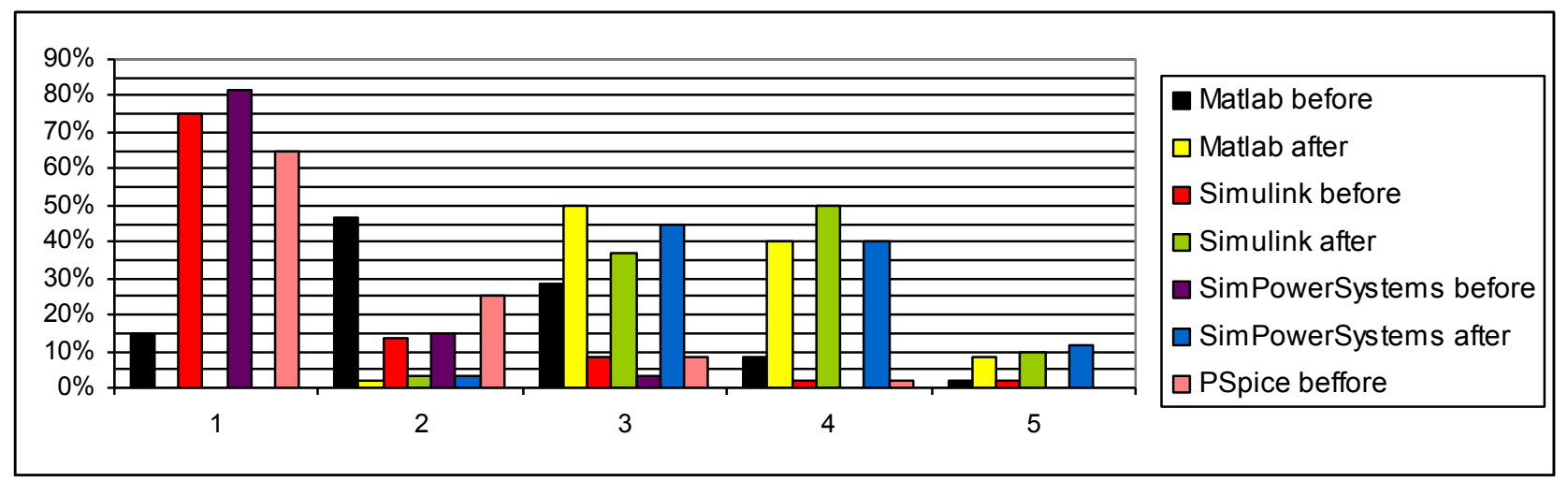

Fig 5. Questionnaire results regarding the knowledge/experience with MATLAB, SIMULINK, SimPowerSystems before and after the course (and also with PSpice before the course)

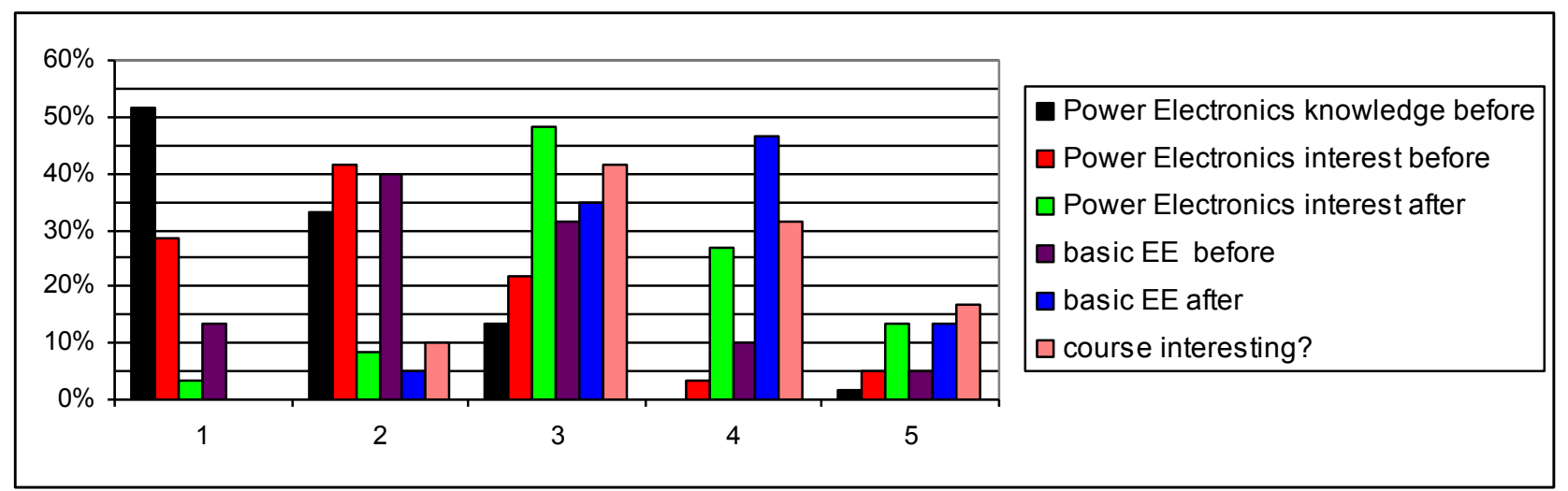

Fig 6. Questionnaire results regarding the students' interest in power electronics and their comprehension of basic Electrical Engineering (EE) topics before and after the course (and the general level of interest of the course as well)

\section{Conclusion}

This paper describes the education scenario followed in the Electrical Engineering Department of the Technological Educational Institute for Crete in 2014 for teaching a first course in power electronics, based on a MATLAB/SIMULINK approach. The syllabus and general teaching approach for the course is briefly described. A 60 questions questionnaire was handed to the students after the completion of the course and results are also briefly described in this paper.

This paper was presented at Pan-Hellenic Conference on Electronics and Telecommunications - PACET, that took place May 8-9 2015, at Ioannina Greece. 


\section{References}

1. OrCAD, http://www.orcad.com/

2. PowerSim, http://powersimtech.com/products/psim/

3. J. Jos Rodrguez, A. Weinstein, P. Lezana, "A Comparison of Power-Electronics Simulation Tools", EE Times, available at: http://www.eetimes.com/document.asp?doc id=1275977

4. PowerGuru, "Power Electronics Design, Simulation, and Analysis Tools", available at http://www.powerguru.org/powerelectronics-design-simulation-analysis-tools

5. Circuits Today, "List of Circuit design / analysis / simulation software" available at: http://www.circuitstoday.com/circuitdesign-and-simulation-softwares
6. GeckoSimulation, GeckoCircuits software, available at: http://www.gecko-simulations.com/geckocircuits.html

7. Plexim, PLECS Simulation Software for Power Electronics, http://www.plexim.com/

8. MathWorks, SimPowerSystems,

http://www.mathworks.com/products/simpower/

9. R. Shaffer, Fundamentals of Power Electronics with MATLAB, Charles River Media, USA, 2006

10. A. K. Tyagi, "MATLAB and SIMULINK for Engineers", Oxford University Press, India, 2012

11. S. Jain, "Modeling \& Simulation using MATLAB-SIMULINK", Wiley India, 2014 